\title{
Mixed integer multi-objective optimization of composite structures with frequency-dependent interleaved viscoelastic damping layers
}

\author{
Chao Xu*, ${ }^{*}$, Miao-Zhang $\mathrm{Wu}^{1}$, Mohamed Hamdaoui ${ }^{2}$ \\ ${ }^{1}$ School of Astronautics, Northwestern Polytechnical University, Xi'an 710072, China \\ ${ }^{2}$ Université de Lorraine, LEM3 UMR 7239, Ile du Saulcy, F-57045 Metz - Cedex 01, France
}

\begin{abstract}
The optimal design of composite structures with frequency-dependent interleaved viscoelastic damping layers is addressed in this paper. The design objective of simultaneously maximizing structural modal damping and frequency and minimizing weight is considered. The design problem is formulated as a mixed integer multi-objective optimization problem and solved by evolutionary algorithm. A layerwise finite element model is used. The Pareto-optimal solutions are obtained for two applications. The results show that the approach is quite useful in integrally designing such kind of composite structures. In particular, it is shown that the inserting position and the material type are two important design parameters.
\end{abstract}

KEYWORDS: viscoelastic damping; mixed integer multi-objective optimization; layerwise finite element; evolutionary optimization; composite structures

* Corresponding author. Tel.: +86 02988493620

E-mail address: chao_xu@nwpu.edu.cn (Chao Xu) 


\section{INTRODUCTION}

Fiber reinforced composites are extensively used in aerospace, civil and automotive industries due to their high specific stiffness, high specific strengths and tailorable anisotropic elastic properties. Although fiber-reinforced composites exhibit higher energy dissipation capacity than common metals, the amount of damping is still not sufficient for many applications especially under severe vibration circumstances [1]. In past decades, a number of works [2-6] to improve the damping of composite structures have been concentrated on inserting viscoelastic damping layers between the base composite laminate. By this hybrid technique, the damping capacity of composite laminate can be increased many times by the shear deformation induced between the soft damping layer and the stiff constraining layer. However, due to interleaving soft material layers within stiff laminate, the mechanical analysis and design optimization of these structures becomes difficult and challenging.

Many works have been done on dynamic analysis of viscoelastic laminated composite structures. For example, DiTaranto and Blasingame [7] proposed analytical solutions for composite viscoelastic beams. Rao et al. [8] presented closed-form solutions for resonance frequencies and modal loss factors of laminated composite beams with multiple viscoelastic layers by the energy formulation and Ritz method. Zhou et al. [9] analytically studied the effects of various geometry and physical parameters on the resonance frequencies and modal loss factors of a composite tube with embedded viscoelastic layers. Finite element method is also used for analyzing viscoelastic sandwich structures by many researchers. Lu et al. [10] compared finite element to experimental results for three layered damped viscoelastic composite plates. Rikards et al. [11] studied the damping features of laminated visco-elastic sandwich beams using finite elements. Rao et al. [12] proposed a finite element based 
modal strain energy method to analyze laminated composite beams with embedded multiple damping layers. Three dimensional solid elements were used to model the viscoelastic material (VEM) and shell elements to discretize the elastic layers in their study. Akoussan et al. [13] elaborated a model for multilayered laminated viscoelastic sandwich plates and proposed a new solution procedure to perform parametric studies of damping characteristics of these structures. Zhang et al. [14] developed a detailed 3D finite element model for predicting the modal loss factor of laminated composite beams with integral viscoelastic layers. The frequency dependence of viscoelastic damping materials was considered. It is concluded that to accurately represent the strain energy induced by the shear of the viscoelastic layer, a full 3-D solid finite element model or a mixed solid/shell composite element model is generally necessary. Although those full 3D or mixed solid/shell finite element models can effectively describe the shear deformation inside the viscoelastic layer, a cumbersome and time consuming mesh generation process are commonly required. Moreover, the developed FE model is not well suited for the optimal design procedure due to the need to completely regenerate three dimensional FE mesh when changing the geometric parameters of damping treatment.

In recent years, Reddy's layerwise theory [15], which is based on a piece-wise description of displacement field, has been applied to develop finite element beam/plate/shell formulations for analyzing viscoelastic laminated structures. Zapfe et al. [16] presented a discrete layer finite element for the dynamics analysis of viscoelastic laminated beams. A discrete layer annular finite element was proposed by Wang et al. [17] for vibration and damping analysis of a three-layered composite annular plate with a viscoelastic mid-layer. Moreira et al. [18] developed a generalized layerwise 
plate finite element for multi-layer viscoelastic damped structures. A full layerwise shell finite element considering frequency dependent material properties was proposed by Oh et al. [19] for cylindrical laminates with viscoelastic layer. Araujo et al. [20] presented a finite element model for composite laminates with frequency dependent viscoelastic core using mixed layerwise approach, by considering a high order shear deformation theory to represent the displacement field of viscoelastic layer and a first order shear deformation theory for the displacement files of adjacent laminated face layers. Ferreira et al. [21] presented a layerwise finite element model based on Carrera's Unified Formulation for the analysis of composite laminated plates with a viscoelastic core.

It can be found that a significant simplification in the spatial finite element modelling of multilayer structures can be achieved by using a layerwise finite element model since it maintains a 2-D type data structure. In addition, the element stiffness matrix can be computed much faster for layerwise element by performing in-plane and out-of-plane integration separately [22]. In this paper, to simplify the modelling and optimization process, a quadrilateral plate finite element based on the layerwise theory is developed for modelling hybrid composite laminates with interleaving viscoelastic layers.

Design optimization is an important procedure to ensure achieving desired structural mechanical and damping capacities for hybrid viscoelastic composite laminates. Some researchers formulate this design problem as a single-objective optimization problem. Araújo et al. [20, 23] conducted a single objective constrained optimization for the maximization of modal loss factor of viscoelastic laminated composite structures, using a gradient based algorithm and mixed layerwise finite element 
model. Montemurro et al. [24] presented a genetic algorithm based optimization technique for the design of damping properties of hybrid elastomer/composite laminates. The only optimization objective was to maximize the first $N$ modal loss factors. Le Maout et al. [25] maximized damping loss factors of a hybrid elastomer/composite sandwich plate taking the total number of layers, their respective thicknesses, their fiber orientations, and the position of the visco-elastic core, and the stacking sentence as design variables. A layerwise optimization method was proposed by Li et al. [26] to design the damping capacity of laminated rectangular plates under general edge conditions using the analytical optimization technique. Montemurro et al. [27] proposed a two-level procedure for the global optimization of the damping behavior of composite laminated plates with elastomer patches.

The presence of soft viscoelastic layers in composite laminate increases the structural damping capacity, while it changes the overall mechanical properties of the composite structure and increases the weight [28]. In real engineering practice, these conflicting design performances need to be considered simultaneously. Therefore, it is natural to formulate a multi-objective optimization problem instead because multi-objective optimization can balance all aspects of design performance and output a set of flexible solutions [29]. Recently, the multi-objective optimization of viscoelastic laminated structures gradually attracts some attention. Multi-objective optimization of viscoelastic laminated composite structures based on genetic algorithm and ABAQUS finite element model was presented by Araújo et al. [23]. Madeira et al. [30, 31] presented a multi-objective method for optimization of viscoelastic laminated sandwich structures for minimizing weight and material cost and maximizing the modal loss factors. The new developed derivative-free algorithm, called direct multi-search (DMS) [50] are used as the optimization tool in their work. Xu et al. [32] proposed a 
multi-objective optimization model for constrained layer damping structures, and only frequencyindependent material properties were considered. Hamdaoui et al. [33] presented a multi-objective optimization approach to choose the most appropriate frequency dependent viscoelastic material for a three-layered rectangular composite beam for low mass and high damping.

In this paper, we present an optimal design method for hybrid composite laminates with interleaved viscoelastic damping layers under general edge conditions using a mixed integer multi-objective optimization method. A quadrangular plate finite element based on the layer-wise theory is developed to model multi-layered hybrid composite laminates under general edge conditions. Frequency-dependent material properties are considered. An evolutionary multi-objective algorithm handling discrete design variables is implemented to find a global optimum. The aim is to obtain designs that give simultaneously low mass, high natural frequency and high modal loss factors. In Section 2, the layer-wise plate finite element model is presented and validated. The optimal design formulation and implementation of the evolutionary multi-objective algorithm are presented in Section 3. In Section 4, the optimization cases are presented. The results are given and discussed in Section 5. 


\section{LAYERWISE FINITE ELEMENT MODELLING}

\subsection{Layerwise plate finite element}

A quadrilateral layerwise plate element is developed and applied to model viscoelastic hybrid multilayered composite laminates. The kinematics is described by the layerwise theory based on the first-order shear deformation assumption in each layer, which was also used in Ref. [34]. Fig.1 represents a section of an $n$-layer hybrid composite laminate. According to the layerwise theory, each layer is modelled as a thick plate fulfilled to the first-order shear deformation theory. The middle surface of the bottom layer is chosen as the reference plane and its displacement components are the reference displacement components. The displacement of the other points on the plate is defined with reference to the displacement of the reference plane.

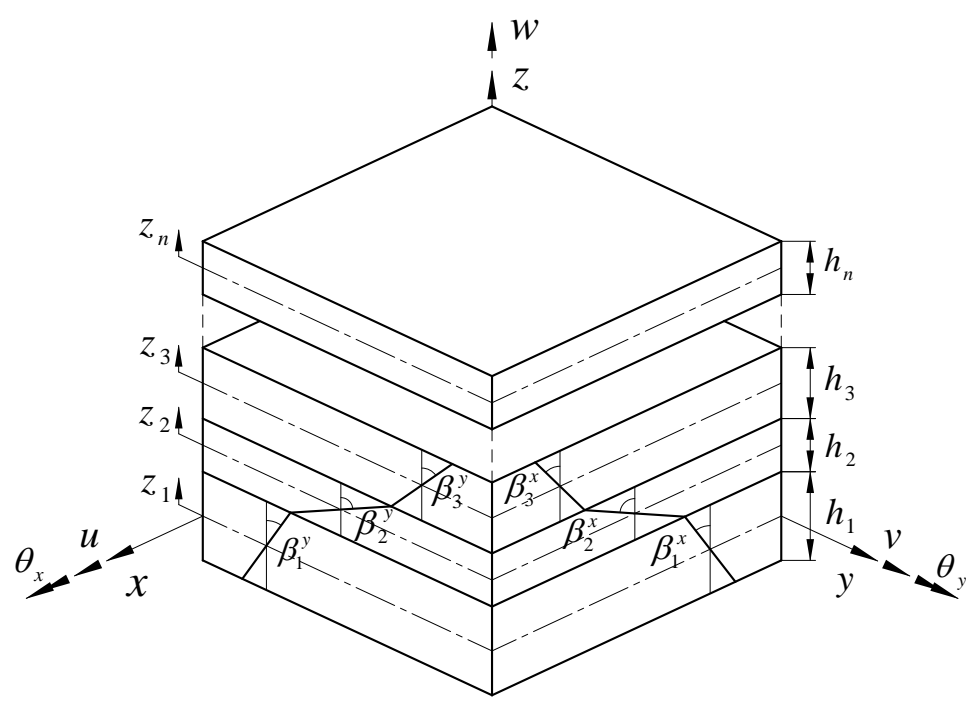

FIG. 1 Kinematic model of the layer-wise theory

Since the first-order shear deformation is assumed in each layer, the in-plane displacement components show a zig-zag behavior through plate thickness. The displacement of each point in the upper layer can be obtained by summing up the displacement of the corresponding point on the 
reference plane and the displacement caused by zig-zag effect, and applying displacement continuity conditions at layer interfaces, defined by

$$
\{\mathbf{u}\}_{k}=\left\{\begin{array}{l}
u_{k} \\
v_{k} \\
w_{k}
\end{array}\right\}=\left\{\begin{array}{c}
u_{0}+\frac{h_{1}}{2} \beta_{1}^{x}+\sum_{j=2}^{k-1} h_{j} \beta_{j}^{x}+\frac{h_{k}}{2} \beta_{k}^{x}+z_{k} \beta_{k}^{x} \\
v_{0}+\frac{h_{1}}{2} \beta_{1}^{y}+\sum_{j=2}^{k-1} h_{j} \beta_{j}^{y}+\frac{h_{k}}{2} \beta_{k}^{y}+z_{k} \beta_{k}^{y} \\
w_{0}
\end{array}\right\}
$$

where $u_{0}, v_{0}$, and $w_{0}$ are displacement components of a point at the reference plane, $\beta^{x}$ and $\beta^{y}$ are the rotations of the normal to the layer mid-plane about the $y$-and $x$-axes, and $h_{\mathrm{k}}$ is the $k$-th layer thicknesses, $z_{k} \in\left[-\frac{h_{k}}{2}, \frac{h_{k}}{2}\right]$,respectively. Based on Eq.1, a generalized displacement field can be defined as

$$
\{d\}=\left\{u_{0}, v_{0}, w_{0}, \beta_{1}^{x}, \beta_{1}^{y}, \cdots, \beta_{k}^{x}, \beta_{k}^{y}, \cdots, \beta_{n}^{x}, \beta_{n}^{y}\right\}^{T}
$$

Eq. 1 can be written into a compact form

$$
\{\mathbf{u}\}_{k}=[T]_{k}\{d\}
$$

with

$$
[T]_{k}=\left[\begin{array}{ccccccccccccc}
1 & 0 & 0 & \frac{h_{1}}{2} & 0 & \ldots & h_{j} & 0 & \ldots & \frac{h_{k}}{2}+z_{k} & 0 & 0 & \ldots \\
0 & 1 & 0 & 0 & \frac{h_{1}}{2} & \ldots & 0 & h_{j} & \ldots & 0 & \frac{h_{k}}{2}+z_{k} & 0 & \ldots \\
0 & 0 & 1 & 0 & 0 & \ldots & 0 & 0 & \ldots & 0 & 0 & 0 & \ldots
\end{array}\right]
$$

The strain field of the $k$-th layer is given by

$$
\{\boldsymbol{\varepsilon}\}_{k}=[L]\{\mathbf{u}\}_{\mathrm{k}}
$$

and the differential operator matrix $[L]$ is

$$
[L]=\left[\begin{array}{ccc}
\frac{\partial}{\partial x} & 0 & 0 \\
0 & \frac{\partial}{\partial y} & 0 \\
\frac{\partial}{\partial y} & \frac{\partial}{\partial x} & 0 \\
\frac{\partial}{\partial z} & 0 & \frac{\partial}{\partial x} \\
0 & \frac{\partial}{\partial z} & \frac{\partial}{\partial y}
\end{array}\right]
$$

The linear elastic constitute relationship is considered for the $k$-th layer, defined by 


$$
\{\boldsymbol{\sigma}\}_{k}=[D]_{k}\{\boldsymbol{\varepsilon}\}_{k}
$$

The stress-strain relation for each layer made of frequency-dependent orthotropic or isotropic material can be written in the form of Eq. (8):

$$
\left\{\begin{array}{l}
\sigma_{11} \\
\sigma_{22} \\
\sigma_{23} \\
\sigma_{13} \\
\sigma_{12}
\end{array}\right\}=\left[\begin{array}{ccccc}
Q_{11}^{*}(i \omega) & Q_{12}^{*}(i \omega) & 0 & 0 & 0 \\
Q_{12}^{*}(i \omega) & Q_{22}^{*}(i \omega) & 0 & 0 & 0 \\
0 & 0 & Q_{44}^{*}(i \omega) & 0 & 0 \\
0 & 0 & 0 & Q_{55}^{*}(i \omega) & 0 \\
0 & 0 & 0 & 0 & Q_{66}^{*}(i \omega)
\end{array}\right]\left\{\begin{array}{c}
\varepsilon_{11} \\
\varepsilon_{22} \\
\varepsilon_{23} \\
\varepsilon_{13} \\
\varepsilon_{12}
\end{array}\right\}
$$

It should be noted that Eq. (8) is written with respect to the principal direction of the layer and the zero transverse normal stress situation is considered. In Eq. (8), $i=\sqrt{-1}, \sigma_{i j}$ are stress components, $\varepsilon_{i j}$ are strain components, and $Q_{i j}^{*}$ are frequency-dependent complex elastic coefficients related to the engineering properties as the follows:

$$
\begin{aligned}
& Q_{11}^{*}(i \omega)=\frac{E_{1}^{*}(i \omega)}{1-v_{12} v_{21}}, Q_{22}^{*}(j \omega)=\frac{E_{2}^{*}(i \omega)}{1-v_{12} v_{21}}, \\
& Q_{12}^{*}(j \omega)=\frac{v_{12} \times E_{2}^{*}(i \omega)}{1-v_{12} v_{21}}, Q_{44}^{*}(i \omega)=G_{23}^{*}(i \omega), \\
& Q_{55}^{*}(i \omega)=G_{31}^{*}(i \omega), Q_{66}^{*}(i \omega)=G_{12}^{*}(i \omega)
\end{aligned}
$$

The frequency dependent complex elastic moduli $E_{1}^{*}, E_{2}^{*}$ and the complex shear moduli $G_{23}^{*}, G_{13}^{*}$ and $G_{12}^{*}$ can be obtained experimentally for a given range of frequencies.

The kinetic energy of the hybrid multi-layer plate structure can be obtained by the sum of the kinetic energy of individual single layer, i.e.,

$$
\Pi^{K}=\sum_{k=1}^{n} \frac{1}{2} \rho_{k} \int_{\Omega_{k}}\{\dot{\mathbf{u}}\}_{k}^{T}\{\dot{\mathbf{u}}\}_{\mathrm{k}} d \Omega_{k}
$$

where $\{\dot{\mathbf{u}}\}_{k}$ denotes the velocity field of the $k$-th layer and $\rho_{k}$ is the mass density. The potential energy of the structure can be calculated by the sum of the potential energy of each individual layer, i.e.,

$$
\Pi^{P}=\sum_{k=1}^{n} \frac{1}{2} \int_{\substack{\Omega_{k} \\ g}}\{\boldsymbol{\sigma}\}_{k}^{T}\{\boldsymbol{\varepsilon}\}_{\mathrm{k}} d \Omega_{k}
$$


As shown in Fig. 2, a four-node isoparametric quadrangular finite element is proposed to discretize the structure. The shape functions of this element in parametric space are

$$
N_{i}=\frac{1}{4}\left(1+\xi_{i} \xi\right)\left(1+\eta_{i} \eta\right) \quad i=1,2,3,4
$$

where $\xi$ and $\eta$ are local parametric coordinates.

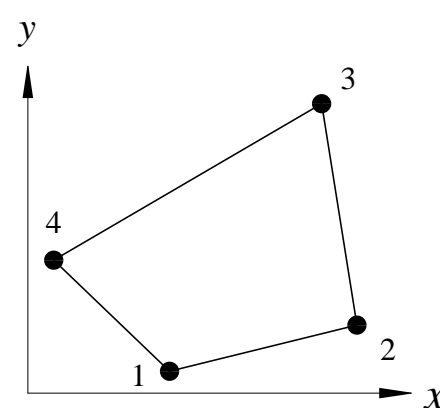

cartesian coordinate

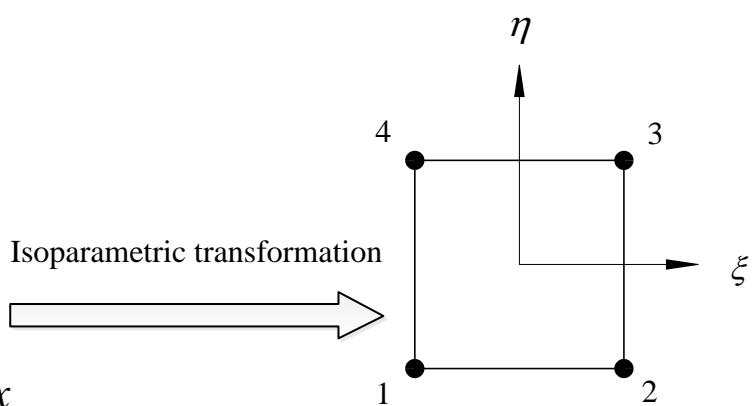

natural coordinate

\section{FIG.2 A four-node quadrangular plate element}

By applying Hamilton's principle, the finite element equations of motion for the hybrid multilayer plate can be deduced as

$$
[M]\{\ddot{U}\}+[K]\{U\}=\{F\}
$$

where $[M]$ and $[K]$ are global mass and stiffness matrices, respectively; $\{U\}$ denotes the vector of global degrees of freedom, and $\{F\}$ is the externally applied mechanical load vector. It is noted that the viscoelastic behavior of the material layer translates into a complex frequency dependent stiffness matrix $[K(i \omega)]$. Assuming harmonic vibrations, and for the free vibration problem, disregarding the excitation terms, Eq. (13) can be reduced to the following complex eigenvalue problem:

$$
\left([K(i \omega)]-\lambda^{*}[M]\right)\left\{\phi^{*}\right\}=\{0\}
$$

where $\left\{\phi^{*}\right\}$ is the complex eigenvector and $\lambda^{*}$ is the complex eigenvalue. 
Note that since a frequency-dependent viscoelastic model is considered in this study, the resulting eigenvalue problem (Eq.14) is nonlinear and hard to be treated numerically. Efficient numerical procedures are required to consider that the global stiffness matrix $[K]$ depends nonlinearly on frequency. In the present study, the complex eigenvalue problem is iteratively solved using the wellknown Implicitly Restarted Arnoldi Method (IRAM) [35] implemented in eigs function of Matlab with shift-invert transformation. The calculated complex eigenvalue can be written as

$$
\lambda^{*}=\lambda^{\prime}+i \lambda^{\prime \prime}
$$

Thus, the circular frequency $\omega$ and the modal loss factor $\eta$ of the hybrid laminate can be calculated by the following formulas:

$$
\omega=\sqrt{\lambda^{\prime}} \text { and } \eta=\frac{\lambda^{\prime \prime}}{\lambda^{\prime}}
$$

\subsection{Model validation}

In this section, to validate the proposed finite element and complex eigenvalue solution procedure, numerical tests with constant and frequency dependent viscoelastic models analyzed in Ref. [36] are calculated. The results by the present analysis are compared to the results given in [36].

The first example is an aluminum/polymer/aluminum beam-plate. The polymer core material is represented by a constant complex modulus. The material and structural data of the sandwich beamplate are listed in Table 1. The core's loss factor is varied as $\eta_{c} \in\{0.1,0.6,1.0,1.5\}$. The present layerwise finite element model is used to investigate its vibration characteristics. The first four vibration modes of the sandwich beam-plate under SFSF and CFFF edge boundary condition (S, simply supported; C, clamped; F, free) for different core material loss factors are given and 
compared to the results obtained in Ref. [36] in Table 2. Note that for comparison, the modal loss factor $\eta_{l}$ is normalized to the input viscoelastic material loss factor $\eta_{c}$.

TABLE 1 Material and structural data of the sandwich beam-plate [36]

\begin{tabular}{|c|c|c|}
\hline Elastic faces & $\begin{array}{l}\text { Young modulus } \\
\text { Poisson ratio } \\
\text { Mass density } \\
\text { Thickness }\end{array}$ & $\begin{array}{l}E_{f}=69000 \mathrm{MPa} \\
v_{f}=0.3 \\
\rho_{f}=2766 \mathrm{kgm}^{-3} \\
H=1.524 \mathrm{~mm}\end{array}$ \\
\hline Viscoelastic polymer core & $\begin{array}{l}\text { Young modulus } \\
\text { Poisson ratio } \\
\text { Mass density } \\
\text { Thickness }\end{array}$ & $\begin{array}{l}E_{c}=1.794 \mathrm{MPa} \\
v_{c}=0.3 \\
\rho_{c}=968.1 \mathrm{kgm}^{-3} \\
h=0.127 \mathrm{~mm}\end{array}$ \\
\hline Sandwich beam-plate & $\begin{array}{l}\text { Length } \\
\text { Width }\end{array}$ & $\begin{array}{l}L=177.8 \mathrm{~mm} \\
l=12.7 \mathrm{~mm}\end{array}$ \\
\hline
\end{tabular}

TABLE 2 Modal frequencies and associated normalized loss factors corresponding to the first four vibration modes of the sandwich beam-plate with constant complex core's modulus and various core's material loss factors

\begin{tabular}{|c|c|c|c|c|c|c|c|c|}
\hline \multirow[t]{2}{*}{$\boldsymbol{\eta}_{c}$} & \multicolumn{2}{|c|}{$\begin{array}{l}\text { Present method } \\
\text { SFSF beam-plate }\end{array}$} & \multicolumn{2}{|c|}{$\begin{array}{c}\text { Ref. [36] } \\
\text { SFSF beam-plate }\end{array}$} & \multicolumn{2}{|c|}{$\begin{array}{l}\text { Present method } \\
\text { CFFF beam-plate }\end{array}$} & \multicolumn{2}{|c|}{$\begin{array}{c}\text { Ref. [36] } \\
\text { CFFF beam-plate }\end{array}$} \\
\hline & $f_{l}(\mathrm{~Hz})$ & $\eta_{l} / \eta_{c}$ & $f_{l}(\mathrm{~Hz})$ & $\eta_{l} / \eta_{c}$ & $f_{l}(\mathrm{~Hz})$ & $\eta_{l} / \eta_{c}$ & $f_{l}(\mathrm{~Hz})$ & $\eta_{l} / \eta_{c}$ \\
\hline \multirow[t]{4}{*}{0.1} & 148.49 & 0.3501 & 148.56 & 0.3500 & 64.3 & 0.281 & 64.3 & 0.281 \\
\hline & 488.37 & 0.1954 & 489.28 & 0.1954 & 297.5 & 0.242 & 298.1 & 0.242 \\
\hline & 1034.39 & 0.1066 & 1038.74 & 0.1066 & 745.7 & 0.154 & 749.0 & 0.153 \\
\hline & 1794.26 & 0.0647 & 1807.57 & 0.0647 & 1396.3 & 0.088 & 1406.9 & 0.088 \\
\hline \multirow[t]{4}{*}{0.6} & 150.69 & 0.3327 & 150.75 & 0.3327 & 65.7 & 0.246 & 65.7 & 0.245 \\
\hline & 489.64 & 0.1940 & 490.56 & 0.1940 & 300.0 & 0.232 & 300.6 & 0.232 \\
\hline & 1035.07 & 0.1064 & 1039.43 & 0.1064 & 747.5 & 0.152 & 750.7 & 0.152 \\
\hline & 1794.67 & 0.0646 & 1807.99 & 0.0646 & 1397.3 & 0.088 & 1407.8 & 0.088 \\
\hline \multirow[t]{4}{*}{1.0} & 154.40 & 0.3051 & 154.47 & 0.3051 & 67.7 & 0.202 & 67.7 & 0.202 \\
\hline & 491.95 & 0.1915 & 492.87 & 0.1914 & 303.9 & 0.217 & 304.5 & 0.217 \\
\hline & 1036.32 & 0.1060 & 1040.69 & 0.1060 & 750.6 & 0.150 & 753.9 & 0.149 \\
\hline & 1795.42 & 0.0645 & 1808.75 & 0.0646 & 1399.0 & 0.087 & 1409.5 & 0.087 \\
\hline \multirow[t]{4}{*}{1.5} & 160.71 & 0.2625 & 160.77 & 0.2625 & 70.1 & 0.153 & 70.2 & 0.153 \\
\hline & 496.37 & 0.1867 & 497.3 & 0.1867 & 310.0 & 0.197 & 310.6 & 0.197 \\
\hline & 1038.75 & 0.1054 & 1043.13 & 0.1054 & 756.4 & 0.145 & 759.7 & 0.145 \\
\hline & 1796.89 & 0.0644 & 1810.23 & 0.0644 & 1402.0 & 0.087 & 1412.6 & 0.086 \\
\hline
\end{tabular}

The second example is a sandwich plate with a core made of 3M ISD 112, whose shear modulus is 
frequency dependent [36]. The material and structural data of the sandwich plate are listed in Table 3.

The frequency-dependent model and properties of $3 \mathrm{M}$ ISD 112 at $27^{\circ} \mathrm{C}$ can be found in appendix.

TABLE 3 Material and structural data of the sandwich plate [36]

\begin{tabular}{|c|c|c|}
\hline Elastic faces & $\begin{array}{l}\text { Young modulus } \\
\text { Poisson ratio } \\
\text { Mass density } \\
\text { Thickness }\end{array}$ & $\begin{array}{l}E_{f}=68900 \mathrm{MPa} \\
v_{f}=0.3 \\
\rho_{f}=2740 \mathrm{kgm}^{-3} \\
H=0.762 \mathrm{~mm}\end{array}$ \\
\hline $\begin{array}{l}\text { Viscoelastic polymer core } \\
3 \mathrm{M} \text { ISD } 112 \text { at } 27^{\circ} \mathrm{C}\end{array}$ & $\begin{array}{l}\text { Poisson ratio } \\
\text { Mass density } \\
\text { Thickness } \\
\text { Shear modulus }\end{array}$ & $\begin{array}{l}\text { see in Appendix } \\
\text { see in Appendix } \\
h=0.254 \mathrm{~mm} \\
\text { see in Appendix }\end{array}$ \\
\hline Sandwich plate & $\begin{array}{l}\text { Length } \\
\text { Width }\end{array}$ & $\begin{array}{l}L=348 \mathrm{~mm} \\
l=304.8 \mathrm{~mm}\end{array}$ \\
\hline
\end{tabular}

TABLE 4 Modal frequencies and associated loss factors corresponding to the first four vibration modes the sandwich plate with $3 \mathrm{M}$ ISD112 frequency dependent core at $27^{\circ} \mathrm{C}$

\begin{tabular}{ccccc}
\hline $\begin{array}{c}\text { Boundary } \\
\text { conditions }\end{array}$ & Present method & \multicolumn{2}{c}{ Ref. [36] } \\
\cline { 2 - 5 } SSSS & $f_{l}(\mathrm{~Hz})$ & $\eta_{l}$ & $f_{l}(\mathrm{~Hz})$ & $\eta_{l}$ \\
& 53.75 & 0.218 & 53.77 & 0.213 \\
& 110.47 & 0.283 & 110.31 & 0.272 \\
& 126.74 & 0.295 & 126.72 & 0.283 \\
CCCC & 176.99 & 0.304 & 176.97 & 0.289 \\
& & & & \\
& 83.08 & 0.254 & 83.01 & 0.246 \\
& 146.83 & 0.267 & 146.61 & 0.258 \\
CSCS & 168.86 & 0.267 & 168.92 & 0.257 \\
& 224.98 & 0.281 & 225.27 & 0.270 \\
& & & & \\
& 66.77 & 0.238 & 66.71 & 0.231 \\
CFCF & 133.68 & 0.294 & 133.66 & 0.282 \\
& 137.40 & 0.265 & 137.11 & 0.280 \\
& 197.01 & 0.292 & 197.13 & 0.218 \\
& & & & 0.209 \\
& 46.74 & 0.223 & 46.62 & 0.238 \\
\hline
\end{tabular}

The first four vibration modes of the sandwich plate are calculated under SSSS, CCCC, CSCS, CFCF edge boundary conditions. Table 4 lists the first four vibration mode frequencies and modal 
loss factors. For comparison, the results given in Ref. [36] are also listed.

It can be observed from Table 2 and Table 4 that the normal frequencies and modal loss factors calculated by the present finite element and solution procedure show good agreement compared to those of published results, providing validation of the proposed layerwise finite element analysis method. 


\section{OPTIMAL DESIGN METHOD}

\subsection{Optimization model}

The main objective of this study is to find the optimal design of a multilayered hybrid composite laminate to ensure achieving high structural damping and satisfactory mechanical performance simultaneously. This kind of design problems are often complex and difficult to solve without considering several conflicting design objectives. For viscoelastic damped composite structures, the maximization of structural modal loss factor is usually considered as a main design objective. Meanwhile, many engineering applications require the control of the added weight to the structures, thus the minimization of structural weight is also considered. In addition, for the hybrid composite laminate with interleaved VEM layers, the minimal decrease of structural stiffness due to inserting soft VEM layers is necessary. Therefore, the objective functions used in this study are stated as follows:

(1) Minimizing the opposite of the sum of modal loss factors of the first $N$ modes. Thus, the first objective function is

$$
\phi_{1}=-\sum_{i=1}^{N} \eta_{i}
$$

(2) Minimizing the total structural mass $m$ of the viscoelastic damping structure. Thus, the second objective function is

$$
\phi_{2}=m
$$

(3) The minimal decrease of stiffness can be expressed as the minimizing the opposite of the sum of modal frequencies of the first $N$ modes, defined as

$$
\phi_{3}=-\sum_{i=1}^{N} f_{i}
$$

It is worth noting that minimizing $\phi_{1}$ or $\phi_{3}$ is equivalent to maximizing $-\phi_{1}$ or $-\phi_{3}$. 
The objective functions $\phi_{1}, \phi_{2}$ and $\phi_{3}$, can be obtained by layerwise finite element analysis and solving the eigenvalue problems of Eq. (14) iteratively, according to a defined frequency dependent complex stiffness matrix and a real mass matrix.

The proposed layerwise finite element analysis is called in the optimization loop. Design variables related to ply material type, stacking sequence, and ply geometry can be considered simultaneously in design optimization. In the practical design optimization of composite laminates, orthotropic ply thickness is often fixed and ply angles are limited to discrete set such as $0^{\circ}, \pm 45^{\circ}$ and $90^{\circ}$ in order to obtain a cost-effective design. Therefore, the viscoelastic material type, the fiber orientation of the orthotropic elastic layer, the inserting position of the VEM layer, and the thickness of VEM layer are taken as design variables in this study. It is noted the variables representing material type, fiber orientation, and VEM layer inserting position are all discrete variables. Since the vibration energy is dissipated through the shear strains induced in the soft VEM layer, the structural damping capacity is sensitive to the variation of the VEM layer thickness. Thus, the variable of the viscoelastic layer thickness is considered as a continuous variable, which can change continuously in a design interval. Hence, the optimization problem is a mixed optimization problem involving naturally continuous and discrete design variables.

In this optimization problem, since the main structural performance indexes are considered as design objectives, constraints are imposed only on some chosen design variables. For the VEM layer thickness variable, constraints are imposed by limiting the upper and lower bound of the variable. For the variable of the inserting position of the viscoelastic layer, the variable must be limited in the 
physical feasible range of the structural geometric boundary.

\subsection{Optimization algorithm}

The formulated multi-objective optimization problem defined above can be written in a more general form as follows,

$$
\begin{aligned}
& \operatorname{minimize} \boldsymbol{\phi}(\boldsymbol{X})=\left[\phi_{1}(\boldsymbol{X}), \phi_{2}(\boldsymbol{X}), \cdots, \phi_{m}(\boldsymbol{X})\right] \\
& \text { subject to } \boldsymbol{X}=\left[x_{1}, x_{2}, \cdots, x_{n}\right] \\
& \qquad x_{i}^{L} \leq x_{i} \leq x_{i}^{U} \quad(i=0,1, \cdots, n)
\end{aligned}
$$

where $\boldsymbol{X}$ is the vector of design variables, $m$ is the number of objectives, $x_{i}^{L}$ and $x_{i}^{U}$ are the lower and upper limits of the design variables, respectively, $n$ is the total number of design variables. It is worth noting that the design variables are either continuous or discrete. For a multi-objective optimization problem, a set of points rather than single optimum is constructed representing tradeoffs between different objectives. The optimal solution of multi-objective optimization is commonly called Pareto front or Pareto surface.

In recent years, global optimization algorithms based on evolutionary computation, which employ principles of natural selection, have been developed to solve multi-objective optimization problems [37-41]. The non-storing genetic algorithm, NSGA-II, is more powerful than the other algorithms since elitism and a crowded comparison operator are used. In this study, a new modified multiobjective genetic algorithm, called reference point based NSGA-II, proposed by Deb et al. in 2006 [42] is used. The main algorithm flowchart of NSGA-II is given in Fig. 3. Suppose the population size is $N$. NSGA-II first generates randomly an initial parent population $P_{\mathrm{t}}$ of size $N$. Then, an 
offspring population $Q_{\mathrm{t}}$ of size $N$ is created from the parent population $P_{\mathrm{t}}$ through genetic operators (selection, crossover and mutation). A new population of size $2 N$ is formed by combining $P_{\mathrm{t}}$ and $Q_{\mathrm{t}}$. The NSGA-II discriminates the combined population by fast non-dominated sorting [39], resulting in a set of non-dominated fronts $F_{1}, F_{2}, \cdots, F_{R}$. Members of the next parent generation $P_{\mathrm{t}+1}$ are selected from solutions in $F_{1}$ preferentially, and then from the second non-dominated front $F_{2}$. The selection process goes on until the size of $P_{\mathrm{t}+1}$ is $N$. Therefore the new population is filled by points of different non-dominated front, one at a time. This method ensures that the optimal solutions, the first non-dominated front $F_{1}$, can be partially kept to the next generation. In generation $t+1$, the offspring population is created from $P_{\mathrm{t}+1}$ and the above process iterates until the maximal number of generations is reached.

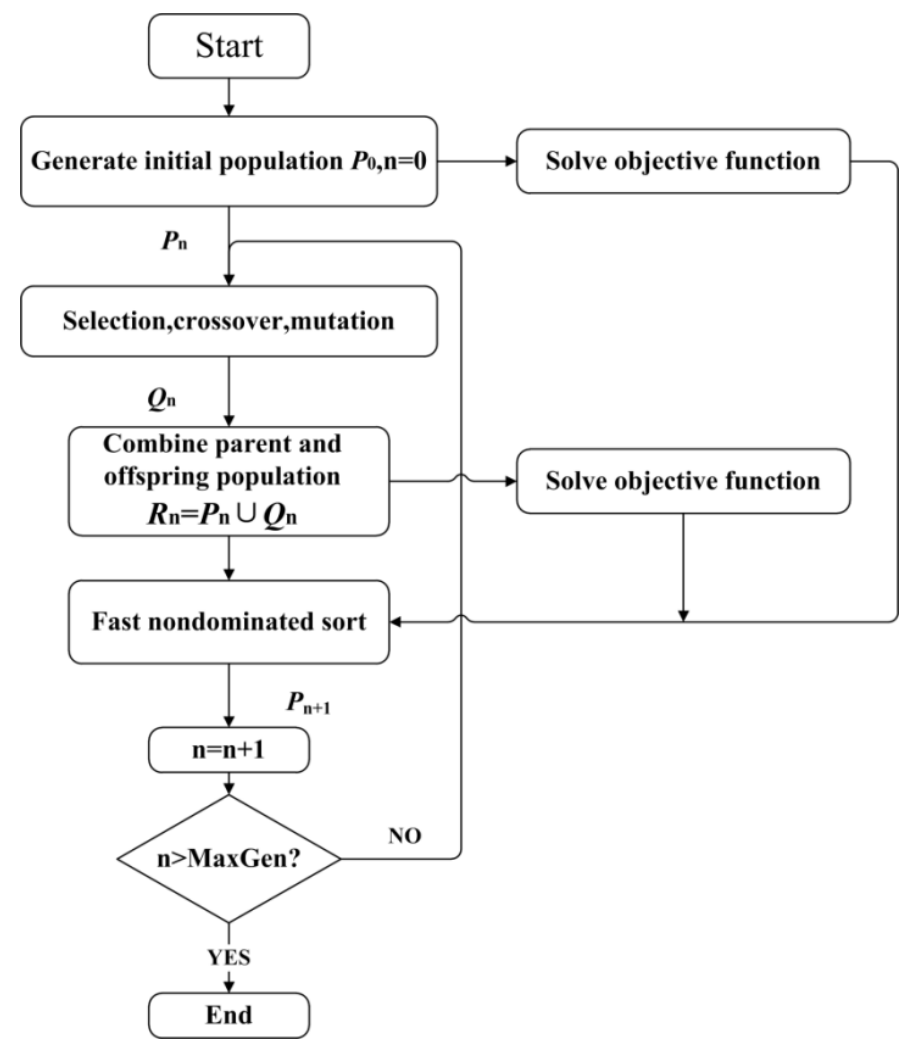

FIG.3 Flowchart of NSGA-II algorithm 
A Matlab $^{R}$ code implementing this algorithm is developed. The code can use real and integer mixed coding. It has the ability of parallel computational evaluations of objective functions, which is very powerful and useful in improving calculation's efficiency. Thanks to parallel computation, the running time has been reduced by $75 \%$ according to the results of numerical examples.

\subsection{Implementation of the optimization algorithm}

In this paper, we consider the optimization of hybrid laminated composites with viscoelastic cores. The design variables considered include the fiber orientation of orthotropic elastic layer, the inserting position of VEM layer, the viscoelastic material type and the thickness of VEM layer. It is noted that both continuous and discrete design variables are included. The classical binary representation is not used here; instead, a mixed integer and real coding strategy is proposed to form the chromosome that represents a population individual.

The encoding of these variables is performed as follows. In this paper, the fiber orientation may take any value from a set of 10 angles from $-45^{\circ}$ and $90^{\circ}$ in steps of $15^{\circ}$. Therefore, the integers from 0 to 9 are used to represent each of the above ply angles. For an $N$-ply laminate, to satisfy the midplane symmetry constraint, only N/2 ply orientations are required to describe the entire laminate. If the symmetrical laminate having odd number plies, the half of the center ply can be considered that it lies on either side of the plane of symmetry. Therefore, the stacking sequence of the laminate is represented by $N / 2$ genes. Each gene is an integer between 0 and 9 . For example, the laminate $\left[90_{2} / 45 /-45 / 90_{2}\right]_{\mathrm{s}}$ is encode as 996099 . The rightmost 9 corresponds to the layer closest to the laminate plane of symmetry. 
The viscoelastic material layer can only be inserted between adjacent orthotropic elastic layers. As shown in Fig.4, for an $\mathrm{N}$-ply composite laminate, due to the mid-plane symmetry constraint, there are only N/2 physical possible positions for inserting viscoelastic material layers. The integers from 1 to N/2 are used to each of these possible positions. The viscoelastic material type is also a discrete variable and coded by integer from 1 to $n$, where $n$ is the number of types of material. The thickness of viscoelastic material layer is a continuous variable and represented by real values between its lower and upper bound.

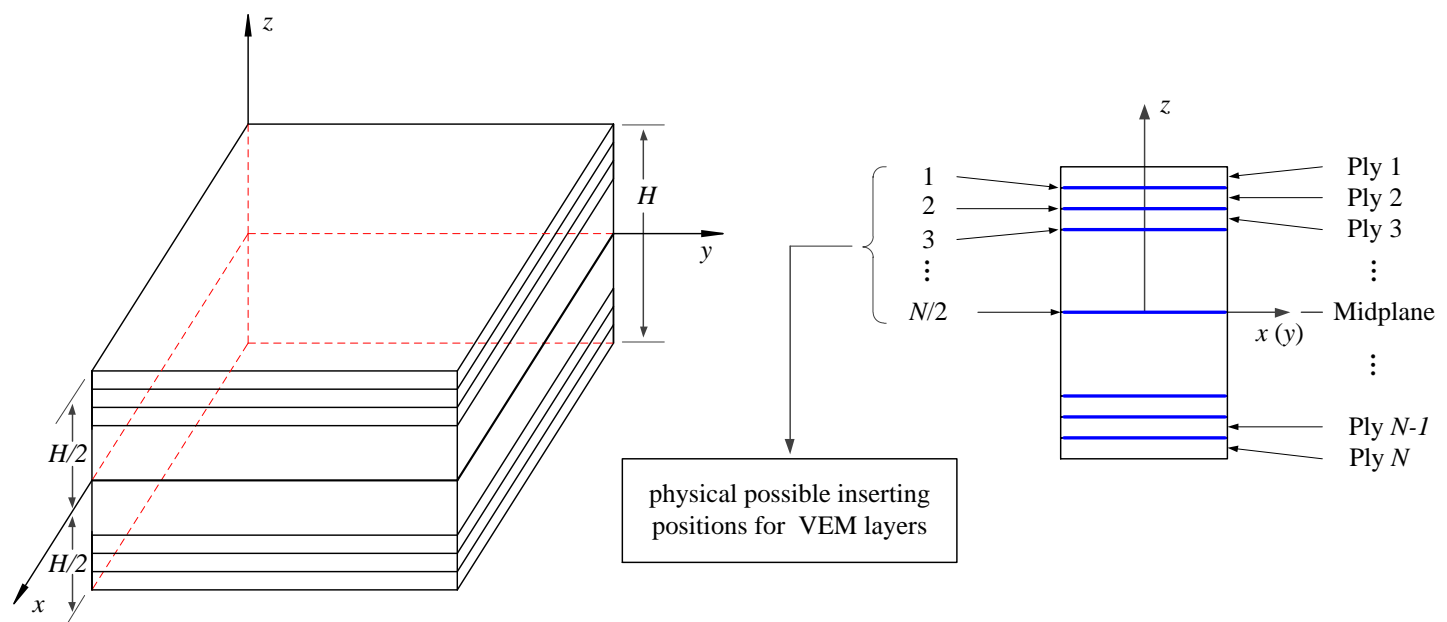

FIG.4 Physical possible positions for inserting viscoelastic material layers

An individual in the population is formed by concatenating the fiber orientation substring with the inserting position substring, the viscoelastic material type substring and the viscoelastic layer thickness substring. This is identified as complete design string. As show in Fig. 5, for an instance, the number of orthotropic elastic plies is 12 . Due to symmetry constraint, a substring consisted of six genes $\left(L_{1} \sim L_{6}\right)$ are used to represent the stacking sequence. A gene is used to represent candidate inserting position of VEM layer. An integer between 1 and 6 is used to represent each physical possible position. A gene is used to represent candidate material type. Six types of viscoelastic 
material are considered and an integer between 1 and 6 is used to code it. A real value is used to represent the thickness of embedded VEM layers. The upper bound is 0.500 and the lower bound is 0.100 .

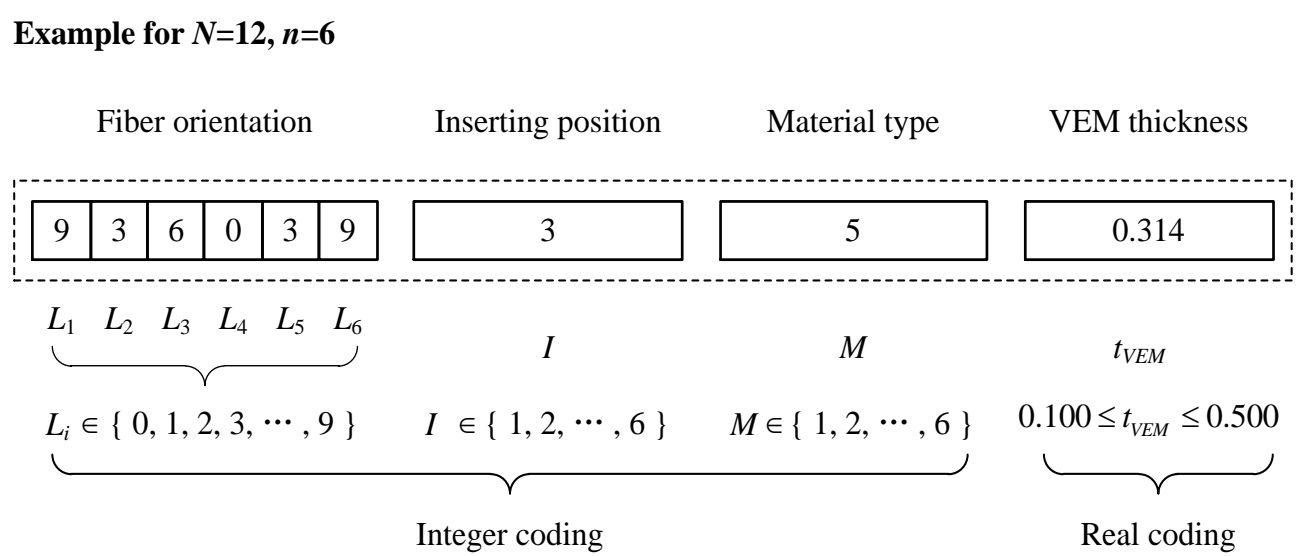

FIG.5 An example of Chromosome representation

The NSGA-II starts with the random generation of a population of design individuals. Then it is processed by means of genetic operators as described in section 3.2. The process is repeated until a stopping criterion is satisfied. The input parameters of the NSGA-II algorithm used in this paper are listed in Table 5.

TABLE 5 Input parameters of NSGA-II

\begin{tabular}{lr}
\hline Population size & Specified by user (default: 100) \\
Maximal number of generation & Specified by user \\
Selection type & Binary tournament selection \\
Crossover type & Arithmetic \\
Crossover fraction & 0.8 \\
Mutation type & Gaussian \\
Mutation fraction & 0.3 \\
\hline
\end{tabular}




\section{OPTIMIZATION CASES}

\subsection{Case 1}

The first case considered here is a symmetrically hybrid composite laminated plate with two interleaved viscoelastic layers. The in-plane dimension of the simply supported sandwich plate is $300 \mathrm{~mm} \times 200 \mathrm{~mm}$. The nominal thickness of each orthotropic ply is $0.25 \mathrm{~mm}$ and the total number of orthotropic plies is fixed to 12 . Thus, the total thickness of the plate without embedded viscoelastic damping layers is $3.00 \mathrm{~mm}$. Two viscoelastic layers are inserted to improve structural damping. The proposed layer-wise finite element model is applied to analyze the laminated composite structure. The sandwich plate is discretized by $16 \times 10$ layer-wise elements. The orthotropic layers are assumed to be graphite/epoxy (GE), and material properties of the unidirectional layer are listed in Table 6[43]. The isotropic viscoelastic material layers are frequency-dependent with an elastic modulus and loss factor referenced from experimental results [44] and characterized by Eq. (21) and (22). In addition, Poisson ratio $\nu=0.49$ and material density $\rho=750 \mathrm{~kg} / \mathrm{m}^{3}$ are used for the viscoelastic material.

$$
\begin{aligned}
\log E_{v} & =0.106 \log f+1.52, E_{v}(\mathrm{MPa}) \\
\eta_{v} & =39.4-5.56 \log f, \eta_{v}(\%)
\end{aligned}
$$

4.2 Case 2

TABLE 6 Material properties of graphite/epoxy unidirectional layer

\begin{tabular}{llll}
\hline$E_{1}$ & $119 \mathrm{GPa}$ & $E_{2}$ & $8.67 \mathrm{GPa}$ \\
$\mu_{12}$ & 0.31 & $G_{12}$ & $5.18 \mathrm{GPa}$ \\
$G_{13}$ & $5.18 \mathrm{GPa}$ & $G_{23}$ & $3.9 \mathrm{GPa}$ \\
$\rho$ & $1570 \mathrm{Kg} / \mathrm{m}^{3}$ & $\eta_{11}$ & $0.118 \%$ \\
$\eta_{22}$ & $0.620 \%$ & $\eta_{12}$ & $0.812 \%$ \\
$\eta_{13}$ & $0.812 \%$ & $\eta_{23}$ & $0.846 \%$ \\
\hline
\end{tabular}


The second case is a three-layered rectangular sandwich beam-plate with a single viscoelastic layer inserted in the middle plane. The in-plane geometry size of the beam is shown in Fig. 6, and the nominal total thickness is $3.175 \mathrm{~mm}$, including the elastic layers and viscoelastic layer. The sandwich beam is modeled using $28 \times 2$ layerwise elements and the boundary condition is of the type clampedfree. The material properties of the isotropic elastic layers are: $E=69 \mathrm{GPa}, v=0.3, \rho=2766 \mathrm{~kg} / \mathrm{m}^{3}$. For the viscoelastic layer in this example, six types of frequency and temperature dependent materials with different damping levels are considered. The detailed material model and properties are listed in Appendix. It is noted that the curve fitting parameters for experimental results of viscoelastic materials are all obtained at room temperature, ranging from $20^{\circ} \mathrm{C}$ to $30^{\circ} \mathrm{C}$. Since the temperature changes are fairly tiny, they are ignored in this study.

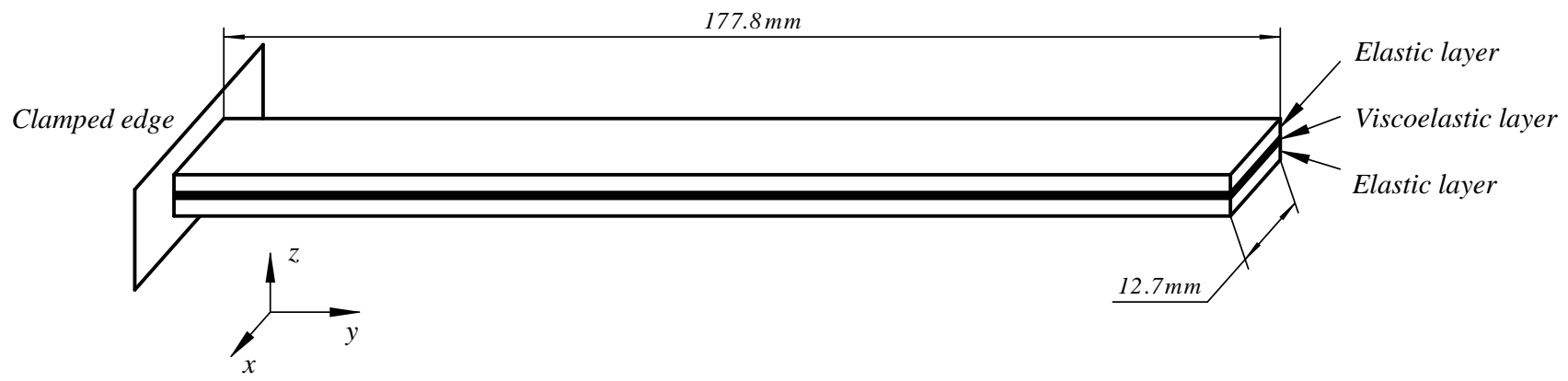

FIG. 6 Geometry of a multi-layer viscoelastic cantilever beam plate 


\section{RESULTS AND DISCUSSIONS}

\subsection{Case 1}

In the first optimal use case, the design objectives are chosen to maximize the first modal frequency and modal loss factor and to minimize the added mass of the structure simultaneously. Since the ply thickness of the fiber reinforced composite is fixed to $0.25 \mathrm{~mm}$, only the fiber angles of the orthotropic layers, the thickness of the embedded viscoelastic layer and the inserting positions of viscoelastic layers are taken as design variables. The thickness of the embedded viscoelastic layer, $t_{\text {vem }}$, varying from $0.1 \mathrm{~mm}$ to $0.5 \mathrm{~mm}$, is encoded by real value. The inserting positions and the fiber angles are both discrete variables and are encoded by decimal integers. Due to symmetry, the integers 1 to 6 are used to encode the six candidate positions for inserting, and only the first six (from bottom to top) layers' ply angles are taken as design variables. It is assumed that the ply angle can vary from $-45^{\circ}$ to $90^{\circ}$ with increment of $15^{\circ}$, and the integers from 0 to 9 are used to encode this variable. The population size in this case is specified as 300 , while maximal number of generations is specified as 100 .

The final three-dimensional Pareto front solutions after 100 generations are displayed in Fig. 7(a), In addition, three different projections of the resulted Pareto non-dominated solutions which are made in $\phi_{1}-\phi_{3}, \phi_{1}-\phi_{2}$, and $\phi_{3}-\phi_{2}$ planes, are depicted in Fig. 7(b), (c) and (d), respectively. In these figures, some specific solutions are labeled and the resulting design configurations are listed in Table 7. In Fig 7(a), the set of the non-dominated solutions form the Pareto-optimal front in three dimensions is depicted. Solution A, B and C are three extreme solutions. Solution A is the best solution for damping $(\eta=10.5 \%)$, but the worst for weight (with $m=45.00 \mathrm{~g}$ ). Solution B is the best 
solution for dynamic stiffness (with $f_{1}=331.27 \mathrm{~Hz}$ ), but the worst for weight too. Solution $\mathrm{C}$ is the best solution for weight (the lightest with $m=9.00 \mathrm{~g})$, but is the worst solution for damping $(\eta=$ $1.39 \%)$

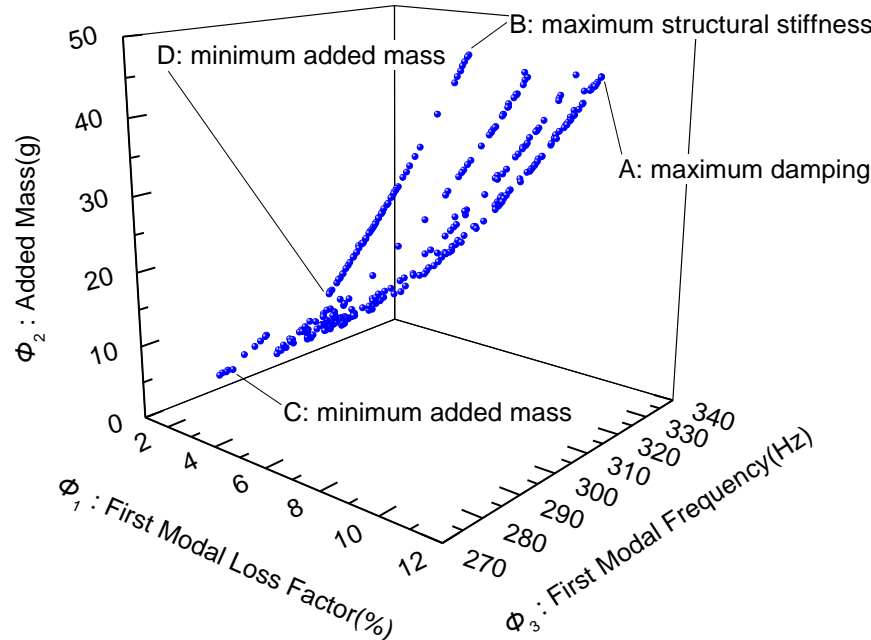

(a)

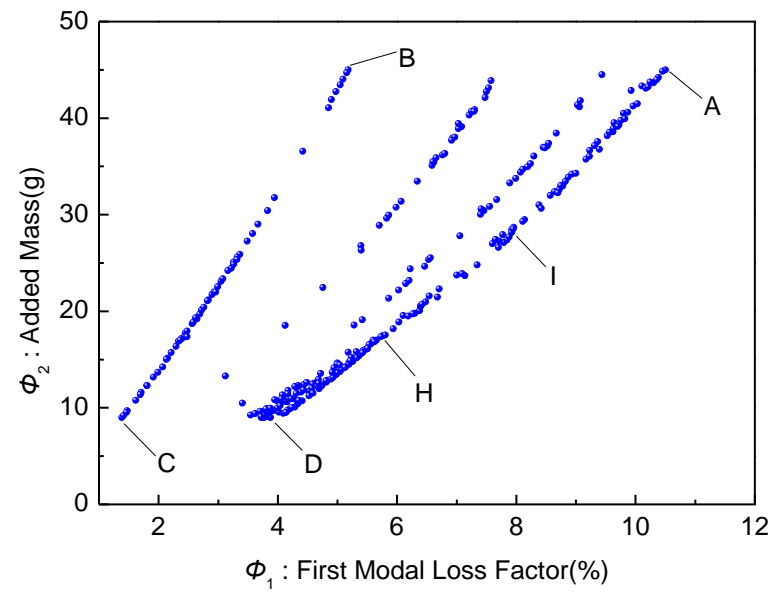

(c)

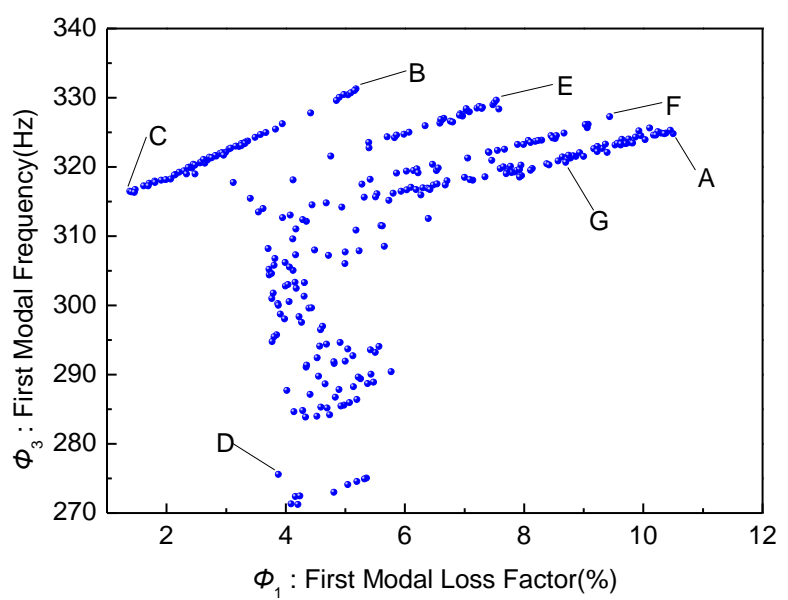

(b)

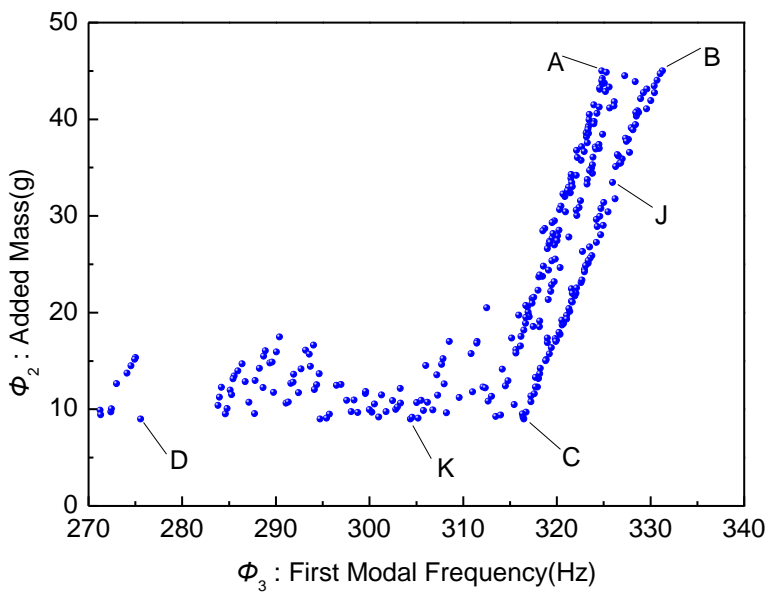

(d)

FIG.7 Pareto front solutions for multi-objective optimization of a laminated sandwich plate: (a) presenting the three-dimensional view; (b), (c) and (d) presenting three plane views.

The Pareto-optimal curves in Fig. 7(b)-(d) enable us to perform trade-off studies. For example, in Fig 7(b), solution $\mathrm{C}$ and $\mathrm{D}$ have identical minimum mass, and the first modal frequency is $14.85 \%$ larger 
than that of D. However, the first modal loss factor of solution $\mathrm{C}$ is $35.82 \%$ of that of solution $\mathrm{D}$. Solution A and solution G are of same stacking sequence except for the thicknesses of VEM layers, but solution A gives both higher loss factor and higher frequency than solution G. Solution C, D and $\mathrm{K}$ all achieve minimum added mass, but the damping and dynamic stiffness capacities are very different due to the different stacking sequence and the inserting positions of the viscoelastic damping layers. In addition, solution E, F, H, I and J correspond to intermediate configurations.

It is noted that the inserting positions have great influence on the dynamic properties of the hybrid structure as expected. For example, if the VEM layers are embedded near the middle plane of the laminated plate, the damped structure obtains higher loss factor. This trend can be verified by simply comparing solution A and solution B, or comparing solution I and solution J.

TABLE 7 Case 1: Pareto-optimal design configurations labeled in Fig. 7

\begin{tabular}{cclccc}
\hline $\begin{array}{c}\text { Optimal } \\
\text { solutions }\end{array}$ & $t_{\text {vem }}$ & \multicolumn{1}{c}{ Stacking sequence } & $\eta_{1}(\%)$ & $m(\mathrm{~g})$ & $f_{1}(\mathrm{~Hz})$ \\
\hline $\mathrm{A}$ & 0.500 & {$\left[90_{5} / 45 / \mathrm{VEM} / \mathrm{VEM} / 45 / 90_{5}\right]$} & 10.50 & 45.00 & 324.80 \\
$\mathrm{~B}$ & 0.500 & {$\left[90_{2} / \mathrm{VEM} / 90 / 75 / 90 / 45 / 45 / 90 / 75 / 90 / \mathrm{VEM} / 90_{2}\right]$} & 5.18 & 45.00 & 331.27 \\
$\mathrm{C}$ & 0.100 & {$\left[90_{2} / \mathrm{VEM} / 90_{2} / 60_{4} / 90_{2} / \mathrm{VEM} / 90_{2}\right]$} & 1.39 & 9.00 & 316.49 \\
$\mathrm{D}$ & 0.100 & {$\left[-30 /-45 / 60 / 45_{2} / \mathrm{VEM} / 15_{2} / \mathrm{VEM} / 45_{2} / 60 /-45 /-30\right]$} & 3.88 & 9.00 & 275.56 \\
$\mathrm{E}$ & 0.479 & {$\left[90_{3} / \mathrm{VEM} / 90_{2} / 45_{2} / 90_{2} / \mathrm{VEM} / 90_{3}\right]$} & 7.54 & 43.14 & 329.63 \\
$\mathrm{~F}$ & 0.494 & {$\left[90_{3} / 75 / \mathrm{VEM} / 75 / 45_{2} / 75 / \mathrm{VEM} / 75 / 90_{3}\right]$} & 9.44 & 44.49 & 327.25 \\
$\mathrm{G}$ & 0.358 & {$\left[90_{5} / 45 / \mathrm{VEM} / \mathrm{VEM} / 45 / 90_{5}\right]$} & 8.70 & 32.26 & 320.65 \\
$\mathrm{H}$ & 0.194 & {$\left[-45_{2} / 45 / 60 / 75 / \mathrm{VEM} / 45_{2} / \mathrm{VEM} / 75 / 60 / 45 /-45_{2}\right]$} & 5.77 & 17.48 & 290.40 \\
$\mathrm{I}$ & 0.316 & {$\left[75 / 90_{3} / 75 / 45 / \mathrm{VEM} / \mathrm{VEM} / 45 / 75 / 90_{3} / 75\right]$} & 7.93 & 28.44 & 318.52 \\
$\mathrm{~J}$ & 0.372 & {$\left[90_{3} / \mathrm{VEM} / 90 / 75 / 30_{2} / 75 / 90 / \mathrm{VEM} / 90_{3}\right]$} & 6.34 & 33.46 & 325.96 \\
$\mathrm{~K}$ & 0.100 & {$\left[-45 / 60_{2} / 75 / 90 / \mathrm{VEM} / 15_{2} / \mathrm{VEM} / 90 / 75 / 60_{2} /-45\right]$} & 3.73 & 9.00 & 304.39 \\
\hline
\end{tabular}

Therefore, from this application it is clear that using a multi-objective optimization method to design frequency dependent composite laminated structure with interleaved viscoelastic layers allows for a great flexibility in the design process. It can handle multiple design objectives simultaneously and 
the resulted non-dominated solutions can by analyzed form several perspectives to give required trade-off designs. The approach presented also provides a novel design tool for the integral dynamic design of those composite structures with hybrid frequency-dependent material layers.

\subsection{Case 2}

The design objectives in the second case are to maximize the first modal frequency and modal loss factor, and to minimize the total mass of the sandwich structure simultaneously. For simplicity, the two isotropic face layers are of same thickness, and the total thickness of the sandwich beam, $t_{\text {nom }}$, is fixed to $3.175 \mathrm{~mm}$. The thickness of the embedded viscoelastic layer, $t_{\text {vem }}$, varying from $0.1 t_{\text {nom }}$ to $0.8 t_{n o m}$, is encoded by a real value. The material type of the viscoelastic core is also taken as design variable. The integers from 1 to 6 , corresponding to the six kinds of viscoelastic materials in Appendix, are used to encode this variable. In this case, the population size is specified as 100 , and maximal number of generations is specified as 100. The final Pareto front solutions after 100 generations are shown in Fig. 8(a) in three dimensions. Three projected views of Pareto front which are made in $\phi_{1}-\phi_{2}, \phi_{1}-\phi_{3}$, and $\phi_{2}-\phi_{3}$ planes, are also shown in Fig. 8(b)-(d). Several Pareto-optimal solutions are presented in Table 8 .

In this application, the aim is to choose the most appropriate viscoelastic material type and best geometric parameters. The results validate that the proposed method is a good tool to perform this task. It is found that some viscoelastic materials, including 3M ISD112 and EAR C-1002, are rejected during the process of optimization. This is because they provide low stiffness, bring more weight or poor damping ability compared to the other materials in this application. By analyzing the 
Pareto-optimal solutions, we can find that the damping levels associated to $\mathrm{ZN}-1$ are better than those associated to PVB, DM 9740 and GE.SMRD materials. However, the solutions associated with ZN-1 material give much lower dynamic stiffness. PVB is actually a kind of weak damping material, it can provide higher structural dynamic stiffness, but the structural damping is dozens of times lower than that of $\mathrm{ZN}-1$. The solutions associated with DM 9740 and GE.SMRD can be regarded as compromises compared to PVB and ZN-1, because both of them provide moderate damping capacity and stiffness. Thus, by the presented method, the most appropriate frequency dependent viscoelastic damping material can be determined.

TABLE 8 Case 2: Pareto-optimal design configurations labeled in Fig. 8

\begin{tabular}{cccccc}
\hline $\begin{array}{c}\text { Optimal } \\
\text { solutions }\end{array}$ & $\begin{array}{c}\text { Viscoelastic } \\
\text { material }\end{array}$ & $t_{\text {vem }}$ & $\eta_{1}(\%)$ & $m(\mathrm{~g})$ & $f_{1}(\mathrm{~Hz})$ \\
\hline $\mathrm{A}$ & ZN-1 & 2.540 & 64.74 & 9.53 & 34.41 \\
$\mathrm{~B}$ & ZN-1 & 2.540 & 64.74 & 9.53 & 34.41 \\
$\mathrm{C}$ & $\mathrm{PVB}$ & 1.661 & 1.03 & 13.20 & 89.70 \\
$\mathrm{D}$ & $\mathrm{PVB}$ & 2.236 & 0.95 & 10.91 & 86.34 \\
$\mathrm{E}$ & PVB & 2.540 & 0.82 & 9.70 & 79.78 \\
$\mathrm{~F}$ & GE.SMRD & 1.108 & 17.84 & 15.66 & 81.29 \\
$\mathrm{G}$ & GE.SMRD & 1.825 & 21.56 & 12.97 & 80.65 \\
$\mathrm{H}$ & GE.SMRD & 2.540 & 17.02 & 10.28 & 72.65 \\
$\mathrm{I}$ & DM 9740 & 1.244 & 7.12 & 15.71 & 85.01 \\
$\mathrm{~J}$ & DM 9740 & 1.783 & 7.92 & 13.93 & 84.16 \\
K & DM 9740 & 2.190 & 7.44 & 12.58 & 80.32 \\
$\mathrm{~L}$ & ZN-1 & 1.386 & 48.80 & 14.21 & 42.33 \\
$\mathrm{M}$ & ZN-1 & 0.318 & 28.49 & 18.54 & 62.63 \\
\hline
\end{tabular}

In addition, for the sandwich configuration interleaved with $\mathrm{ZN}-1$, the larger is the viscoelastic material layer thickness, the larger the structural damping. The relationship between them is monotonic. While for the sandwich configuration inserted with DM 9740 or GE.SMRD, the damping and frequency shows a non-monotonic variation with the thickness variation of the viscoelastic layer. After the inserting material type is chosen, the Pareto-optimal material thickness can easily found 
according to the compromise of the three design objectives. Therefore, simultaneously determining the optimal material type and geometric parameters is achieved.

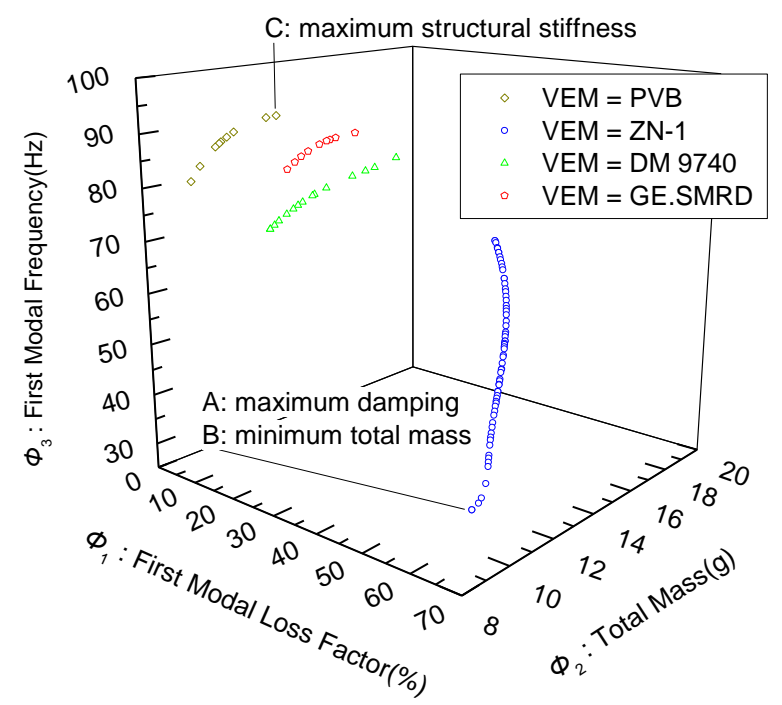

(a)

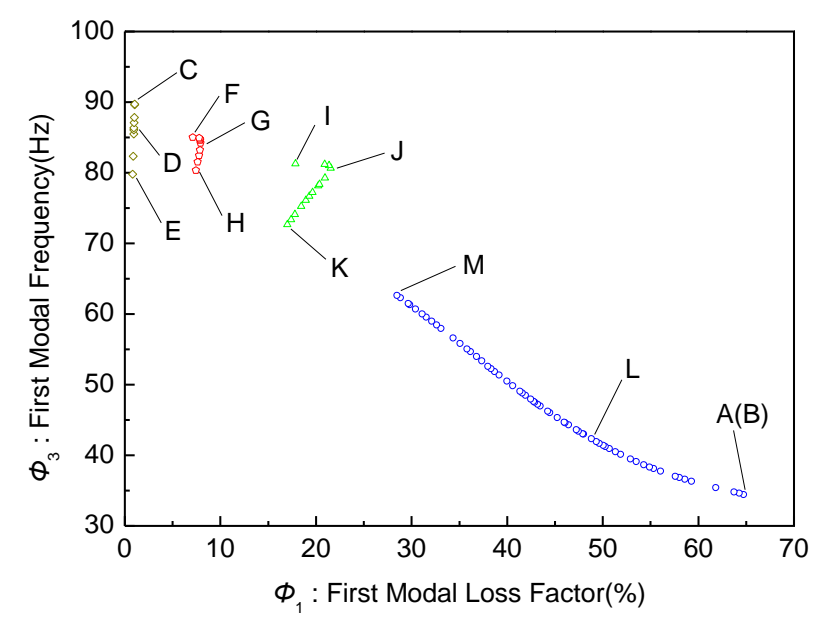

(c)

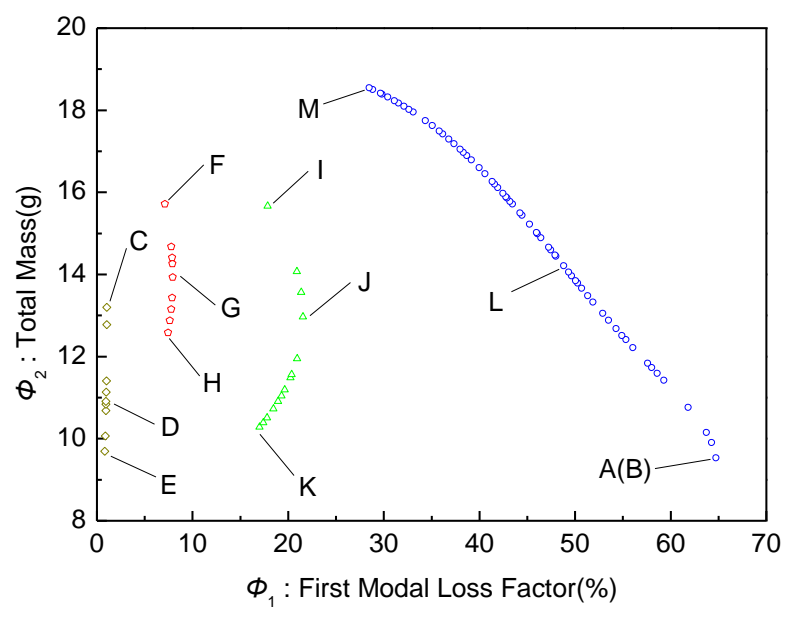

(b)

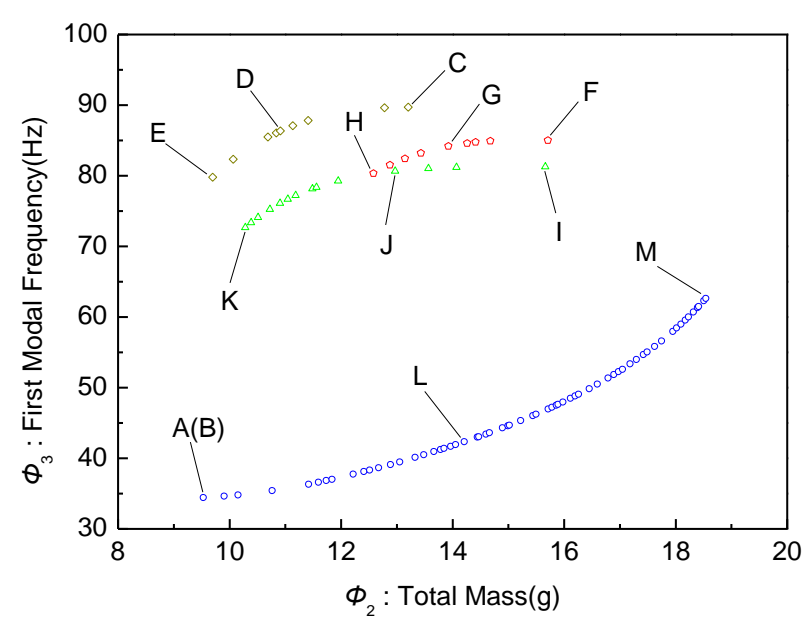

(d)

FIG.8 Pareto-optimal solutions for multi-objective optimization of a viscoelastic sandwich beam: (a) presenting the three-dimensional view; (b), (c) and (d) presenting three plane views. 


\section{CONCLUSIONS}

In this paper, a multi-objective approach to the optimal design of composite structures with frequency-dependent interleaved viscoelastic layers for simultaneous high damping, low added mass and high dynamic stiffness has been presented. Layerwise plate finite element analysis is proposed and applied in the optimization loop. The design problem is formulated as a mixed integer multiobjective optimization problem. An efficient evolutionary algorithm, NSGA-II, is introduced to solve this optimization problem. Parallel computation is applied to improve the computational efficiency.

Non-dominated solutions have been obtained for a general composite plate with two interleaved viscoelastic damping layers and a sandwich beam with one inserting viscoelastic damping layer. In the first application, the Pareto-optimal design solutions with the laminate staking sequence, damping inserting position and thickness as design variables are determined. In the second application, the most appropriate frequency dependent viscoelastic damping material type and its geometric parameter are determined simultaneously. Numerical applications validated the effectiveness of the proposed method and the obtained results can be very useful in the design such kind of composite structures. 


\section{ACKNOWLEDGMENT}

The present work was partially supported by National Science Foundation of China (No.11372246) and NSAF (No.U1530139). 


\section{APPENDIX}

Models and frequency-dependent properties of viscoelastic materials at reference temperature used in this study.

\begin{tabular}{|c|c|c|c|c|c|}
\hline & Material & Complex modulus $G^{*}(\omega)=$ & Fitting parameters & $\begin{array}{c}\text { Reference } \\
\text { Temperature }\end{array}$ & Other properties \\
\hline 1 & $\operatorname{PVB}[33,45]$ & $G_{\infty}+\left(G_{0}-G_{\infty}\right)\left(1+(i \omega \tau)^{1-a}\right)^{-b}$ & $\begin{array}{l}G_{0}=0.479 \mathrm{MPa}, G_{\infty}=235 \mathrm{MPa}, \\
\tau=0.3979 \mathrm{~s}, a=0.46, \mathrm{~b}=0.1946\end{array}$ & $20^{\circ} \mathrm{C}$ & $\begin{array}{c}v=0.49 \\
\rho=999 \mathrm{Kg} / \mathrm{m}^{3}\end{array}$ \\
\hline 2 & $\mathrm{ZN}-1[46]$ & $G_{0}\left(1+\sum_{k=1}^{k=3} \hat{\alpha}_{k} \frac{(i \omega)^{2}+2 \widehat{\zeta}_{k} \widehat{\omega}_{k} i \omega}{(i \omega)^{2}+2 \widehat{\zeta}_{k} \widehat{\omega}_{k} i \omega+\widehat{\omega}_{k}^{2}}\right)$ & $\begin{array}{c}G_{0}=0.5542 \mathrm{MPa}, \\
\hat{\alpha}_{1}=3.960, \hat{\zeta}_{1}=148.0, \widehat{\omega}_{1}=896200 \mathrm{rad} / \mathrm{s} \\
\hat{\alpha}_{2}=65.69, \hat{\zeta}_{2}=12.16, \widehat{\omega}_{2}=927800 \mathrm{rad} / \mathrm{s} \\
\hat{\alpha}_{3}=1.447, \hat{\zeta}_{3}=810.4, \widehat{\omega}_{3}=716300 \mathrm{rad} / \mathrm{s}\end{array}$ & $30^{\circ} \mathrm{C}$ & $\begin{array}{c}v=0.49 \\
\rho=970 \mathrm{Kg} / \mathrm{m}^{3}\end{array}$ \\
\hline 3 & 3M ISD112[47] & \multirow{2}{*}{$G_{0}\left(1+\sum_{k=1}^{k=3} \frac{\Delta_{k} \omega}{\omega-i \Omega_{k}}\right)$} & $\begin{array}{c}G_{0}=0.5 \mathrm{MPa} \\
\Delta_{1}=0.746, \Omega_{1}=468.7 \mathrm{rad} / \mathrm{s} \\
\Delta_{2}=3.265, \Omega_{2}=4742.4 \mathrm{rad} / \mathrm{s} \\
\Delta_{3}=43.284, \Omega_{3}=71532.5 \mathrm{rad} / \mathrm{s}\end{array}$ & $27^{\circ} \mathrm{C}$ & $\begin{array}{c}v=0.5 \\
\rho=1600 \mathrm{Kg} / \mathrm{m}^{3}\end{array}$ \\
\hline 4 & DM 9740[48] & & $\begin{array}{c}G_{0}=1.4 \mathrm{MPa} \\
\Delta_{1}=7.262, \Omega_{1}=406.611 \mathrm{rad} / \mathrm{s} \\
\Delta_{2}=288181.234 \\
\Omega_{2}=22490029.8 \mathrm{rad} / \mathrm{s} \\
\Delta_{3}=2.039, \Omega_{3}=46.750 \mathrm{rad} / \mathrm{s}\end{array}$ & $\begin{array}{c}\text { Room } \\
\text { Temperature }\end{array}$ & $\begin{array}{c}v=0.49 \\
\rho=1101 \mathrm{Kg} / \mathrm{m}^{3}\end{array}$ \\
\hline $5^{\mathrm{a}}$ & GE.SMRD[49] & \multirow{2}{*}{$G_{0}+G_{0}(d-1) \frac{(i \omega \tau)^{\alpha}}{(1+i \omega \tau)^{\beta}}$} & $\begin{array}{c}G_{0}=5.0 \mathrm{MPa}, \mathrm{d}=36 \\
\alpha=0.605, \beta=0.554, \tau=2.09 \times 10^{-4} \mathrm{~s}\end{array}$ & $29^{\circ} \mathrm{C}$ & $\begin{array}{c}\nu=0.49 \\
\rho=1300 \mathrm{Kg} / \mathrm{m}^{3}\end{array}$ \\
\hline 6 & $\begin{array}{c}\text { EAR C-1002 } \\
{[30,49]}\end{array}$ & & $\begin{array}{c}G_{0}=0.8 \mathrm{MPa}, \mathrm{d}=1570, \\
\alpha=0.566, \beta=0.558, \tau=7.23 \times 10^{-10} \mathrm{~s}\end{array}$ & $25^{\circ} \mathrm{C}$ & $\begin{array}{c}\nu=0.49 \\
\rho=1300 \mathrm{Kg} / \mathrm{m}^{3}\end{array}$ \\
\hline
\end{tabular}

${ }^{\text {a }}$ The density of GE.SMRD is estimated. 


\section{REFERENCES}

1. Finegan IC, Gibson RF. Recent research on enhancement of damping in polymer composites. Compos Struct 1999;44:89-98.

2. Rotz C. Cocured damping layers in composite structures. Adv Mater Process 1991;23:352-63.

3. Maly JR, Johnson CD. Cocured viscoelastic composites. 1996 Symp. Smart Struct. Mater., International Society for Optics and Photonics; 1996, p. 365-76.

4. Napolitano KL, Grippo W, Kosmatka JB, Johnson CD. A comparison of two cocured damped composite torsion shafts. Compos Struct 1998;43:115-25.

5. Biggerstaff JM, Kosmatka JB. Damping performance of cocured composite laminates with embedded viscoelastic layers. 5th Annu. Int. Symp. Smart Struct. Mater., International Society for Optics and Photonics; 1998, p. 107-14.

6. Robinson MJ, Kosmatka JB. Embedding viscoelastic damping materials in low-cost VARTM composite structures. Smart Struct. Mater., International Society for Optics and Photonics; 2005, p. 349-60.

7. DiTaranto RA, Blasingame W. Composite damping of vibrating sandwich beams. J Eng Ind 1967;89:633-8.

8. Rao MD, He S. Dynamic analysis and design of laminated composite beams with multiple damping layers. AIAA J 1993;31:736-45.

9. Zhou H, Rao MD. Damping of composite tubes with embedded viscoelastic layers. J Vib Acoust 1996;118:384-9.

10. Lu YP, Killian JW, Everstine GC. Vibrations of three layered damped sandwich plate composites. J Sound Vib 1979;64:63-71. 
11. Rikards R, Chate A, Barkanov E. Finite element analysis of damping the vibrations of laminated composites. Comput Struct 1993;47:1005-15.

12. Rao MD, Echempati R, Nadella S. Dynamic analysis and damping of composite structures embedded with viscoelastic layers. Compos Part B Eng 1997;28:547-54.

13. Akoussan K, Boudaoud H, Daya E-M, Carrera E. Vibration Modeling of Multilayer Composite Structures with Viscoelastic Layers. Mech Adv Mater Struct 2015;22:136-49.

14. Zhang SH, Chen HL. A study on the damping characteristics of laminated composites with integral viscoelastic layers. Compos Struct 2006;74:63-9.

15. Reddy JN. Mechanics of laminated composite plates: theory and analysis. CRC press; 1997.

16. Zapfe JA, Lesieutre GA. A discrete layer beam finite element for the dynamic analysis of composite sandwich beams with integral damping layers. Comput Struct 1999;70:647-66.

17. Wang H-J, Chen L-W. Vibration and damping analysis of a three-layered composite annular plate with a viscoelastic mid-layer. Compos Struct 2002;58:563-70.

18. Moreira RAS, Rodrigues JD, Ferreira AJM. A generalized layerwise finite element for multilayer damping treatments. Comput Mech 2006;37:426-44.

19. Oh IK. Damping characteristics of cylindrical laminates with viscoelastic layer considering temperature-and frequency-dependence. J Therm Stress 2008;32:1-20.

20. Araújo AL, Soares CMM, Soares CAM, Herskovits J. Optimal design and parameter estimation of frequency dependent viscoelastic laminated sandwich composite plates. Compos Struct 2010;92:2321-7.

21. Ferreira AJM, Araújo AL, Neves AMA, Rodrigues JD, Carrera E, Cinefra M, et al. A finite element model using a unified formulation for the analysis of viscoelastic sandwich laminates. 
Compos Part B Eng 2013;45:1258-64.

22. Robbins DH, Reddy JN. Modelling of thick composites using a layerwise laminate theory. Int $\mathrm{J}$ Numer Methods Eng 1993;36:655-77.

23. Araújo AL, Martins P, Soares CMM, Soares CAM, Herskovits J. Damping optimization of viscoelastic laminated sandwich composite structures. Struct Multidiscip Optim 2009;39:569_ 79.

24. Montemurro M, Koutsawa Y, Belouettar S, Vincenti A, Vannucci P. Design of damping properties of hybrid laminates through a global optimisation strategy. Compos Struct 2012;94:3309-20.

25. Le Maout N, Verron E, Bègue J. Simultaneous geometrical and material optimal design of hybrid elastomer/composite sandwich plates. Compos Struct 2011;93:1153-7.

26. Li J, Narita Y. Analysis and optimal design for the damping property of laminated viscoelastic plates under general edge conditions. Compos Part B Eng 2013;45:972-80.

27. Montemurro M, Vincenti A, Koutsawa Y, Vannucci P. A two-level procedure for the global optimization of the damping behavior of composite laminated plates with elastomer patches. J Vib Control 2015;21:1778-800.

28. Lepoittevin G, Kress G. Effect of the Thickness and Position of Soft Layers in Composite Laminates on the Bending Stiffness. Mech Adv Mater Struct 2013;20:497-503.

29. Madeira JFA, Araújo AL, Soares CMM, Soares CAM. Multiobjective optimization of viscoelastic laminated sandwich structures using the Direct MultiSearch method. Comput Struct 2015;147:229-35.

30. Madeira JFA, Araújo AL, Soares CMM, Soares CAM, Ferreira AJM. Multiobjective design of 
viscoelastic laminated composite sandwich panels. Compos Part B Eng 2015;77:391-401.

31. Marler RT, Arora JS. Survey of multi-objective optimization methods for engineering. Struct Multidiscip Optim 2004;26:369-95.

32. Xu C, Lin S, Yang Y. Optimal design of viscoelastic damping structures using layerwise finite element analysis and multi-objective genetic algorithm. Comput Struct 2015;157:1-8.

33. Hamdaoui M, Robin G, Jrad M, Daya EM. Optimal design of frequency dependent threelayered rectangular composite beams for low mass and high damping. Compos Struct 2015;120:174-82.

34. Moreira RAS, Rodrigues JD. A layerwise model for thin soft core sandwich plates. Comput Struct 2006;84:1256-63.

35. Radke RJ. A Matlab implementation of the implicitly restarted Arnoldi method for solving large-scale eigenvalue problems. Diss. Rice University, 1996.

36. Bilasse M, Azrar L, Daya EM. Complex modes based numerical analysis of viscoelastic sandwich plates vibrations. Comput Struct 2011;89:539-55.

37. Schaffer JD. Multiple objective optimization with vector evaluated genetic algorithms. Proc. 1st Int. Conf. Genet. Algorithms, L. Erlbaum Associates Inc.; 1985, p. 93-100.

38. Fonseca CM, Fleming PJ. Multiobjective genetic algorithms. Genet. algorithms Control Syst. Eng. IEE Colloq., IET; 1993, p. 1-6.

39. Srinivas N, Deb K. Muiltiobjective optimization using nondominated sorting in genetic algorithms. Evol Comput 1994;2:221-48.

40. Zitzler E, Thiele L. Multiobjective evolutionary algorithms: a comparative case study and the strength Pareto approach. Evol Comput IEEE Trans 1999;3:257-71. 
41. Deb K, Pratap A, Agarwal S, Meyarivan T. A fast and elitist multiobjective genetic algorithm: NSGA-II. Evol Comput IEEE Trans 2002;6:182-97.

42. Deb K, Sundar J. Reference point based multi-objective optimization using evolutionary algorithms. Proc. 8th Annu. Conf. Genet. Evol. Comput., ACM; 2006, p. 635-42.

43. Manconi E, Mace BR. Estimation of the loss factor of viscoelastic laminated panels from finite element analysis. J Sound Vib 2010;329:3928-39.

44. Berthelot J-M, Sefrani Y. Damping analysis of unidirectional glass fiber composites with interleaved viscoelastic layers: Experimental investigation and discussion. J Compos Mater 2006;40:1911-32.

45. Haberman MR. Design of high loss viscoelastic composites through micromechanical modeling and decision based materials design. Diss. Georgia Institute of Technology, 2007.

46. Zhang L, Du H, Shi Y, Shi X. Parametric determination for GHM of ZN-1 viscoelastic material. Rare Met Mater Eng 2002;31:92-5.

47. Trindade MA, Benjeddou A, Ohayon R. Modeling of frequency-dependent viscoelastic materials for active-passive vibration damping. J Vib Acoust 2000;122:169-74.

48. Wang Y, Inman DJ. Finite element analysis and experimental study on dynamic properties of a composite beam with viscoelastic damping. J Sound Vib 2013;332:6177-91.

49. Pritz T. Five-parameter fractional derivative model for polymeric damping materials. J Sound Vib 2003;265:935-52.

50. Custódio AL, Madeira JFA, Vaz AIF, Vicente LN. Direct multisearch for multiobjective optimization. SIAM J Optim 2011;21:1109-40. 